\title{
Combined transurethral and laparoscopic partial cystectomy for the treatment of bladder endometriosis
}

\author{
Fahad Algreisi ${ }^{1 *}$, Hattan Badr ${ }^{2}$, Daniya Alturkistani ${ }^{3}$, George Vilos ${ }^{4}$
}

\author{
${ }^{1}$ Department of Gynecology, ${ }^{2}$ Department of Urology, King Abdullah Medical City, The Holy Capital (KAMC-HC), \\ Makkah, Saudi Arabia \\ ${ }^{3}$ Department of Medicine, Umm Al-Qura University, Makkah, Saudi Arabia \\ ${ }^{4}$ Department of Obstetrics and Gynecology, Western University, London, Canada
}

Received: 30 January 2017

Revised: 10 February 2017

Accepted: 11 February 2017

\author{
*Correspondence: \\ Dr. Fahad Algreisi, \\ E-mail: Fahadalgreisi@gmail.com
}

Copyright: (C) the author(s), publisher and licensee Medip Academy. This is an open-access article distributed under the terms of the Creative Commons Attribution Non-Commercial License, which permits unrestricted non-commercial use, distribution, and reproduction in any medium, provided the original work is properly cited.

\begin{abstract}
We present a case of a 22-year-old nulliparous woman, initially investigated by a urologist after she presented with a history of urinary tract symptoms including catamenial hematuria and suprapubic pain. Ultrasonographic and cystoscopic findings suggested a bladder mass suspicious for endometriosis. Further MRI revealed a $3 \mathrm{X} 4 \mathrm{~cm}$ mass in the bladder, and the diagnosis of endometriosis was confirmed by the biopsy. Consequently, the patient was offered treatment options including combined laparoscopic and transurethral resection of the bladder lesion for definitive diagnosis and treatment. Using a combination of hydrodissection and $\mathrm{CO}_{2}$ laser energy laparoscopically and monopolar electro-surgery cystoscopically, the lesion was resected uneventfully and the bladder defect was repaired laparoscopically. The patient was discharged on the same day with a Foley catheter, which was removed 10 days later. After 8 weeks follow up period, she remained free of symptoms. Histopathology confirmed endometriosis. We conclude that this combined approach is feasible, safe and effective therapy for intramural bladder endometriosis.
\end{abstract}

Keywords: Bladder, Endometriosis, Laparoscopy, Partial cystectomy, Transurethral

\section{INTRODUCTION}

Endometriosis is a common gynecological disease that affects $15 \%$ of premenopausal women. ${ }^{1}$ Deep infiltrative endometriosis, however, involves a more serious invasion of uterus, posterior fornix, uterosacral ligament, rectum, vagina and urinary tract. ${ }^{2}$ Involvement of the bladder with a penetrating lesion occurs in less than $2 \%$ of all cases of endometriosis. ${ }^{1}$ The condition is often accompanied by pelvic and supra pubic pain, frequency, urgency, nocturia, and less frequently; hematuria. Those common symptoms of vesical endometriosis were also reported in many other diseases of the lower urinary tract, such as interstitial cystitis and chronic urethral syndrome that often complicates the diagnosis. ${ }^{2,3}$ The most effective treatment for bladder endometriosis is a complete surgical resection of the nodule followed by bladder reconstruction. $^{1}$ We present a case of vesical endometrioma managed by a combined procedure of laparoscopic and transurethral partial cystectomy, followed by laparoscopic bladder reconstruction.

\section{CASE REPORT}

A 22-year-old nulliparous woman was referred to us from her urologist with a history of urinary tract symptoms consisting of cyclical hematuria and supra pubic pain, with incidental findings during investigating cystoscopy 
and an ultrasound of a mass suspected for endometriosis. She was otherwise healthy. Physical examination was unremarkable. A diagnosis of endometriosis was made based on her pelvic MRI; which revealed a $3 \times 4 \mathrm{~cm}$ mass inside the bladder, and the histopathological findings of the biopsied specimen. A second look cystoscopy performed by the urologist showed a lesion at the dome of the bladder, with both ureteral orifices being free from endometriosis (Figure 1).

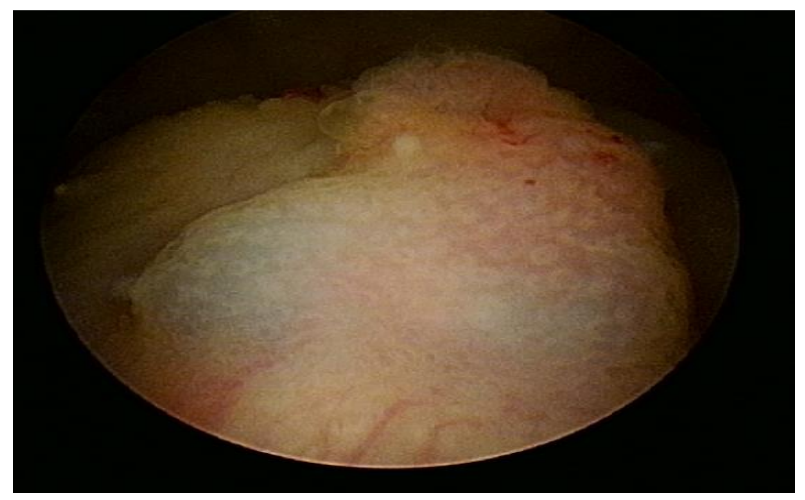

Figure 1: Cystoscopy view of bladder endometriosis.

The laparoscopic panoramic view of the pelvis disclosed negative findings for the presence of any mass in the culde-sac and ovaries. For her concurrent bladder symptoms, she was offered laser laparoscopy and transurethral resection of the bladder lesion. Risks and benefits of the surgery were explained to the patient and formal consent was obtained. A combined procedure of laparoscopic and transurethral resection of the lesion was selected as the surgical approach for this patient. Following bilateral ureteric stenting to ensure the safety of the ureters, a Foley catheter was inserted and the laparosopic surgery was performed. Three trocars were inserted, a $10 \mathrm{~mm}$ at the umbilicus, and two others $5 \mathrm{~mm}$ each on either side of the umbilicus, using the entry technique described by Vilos et al. ${ }^{4}$ The bladder was densely adherent to the anterior wall of the uterus and the bladder lesion was visualized and documented (Figure 2).

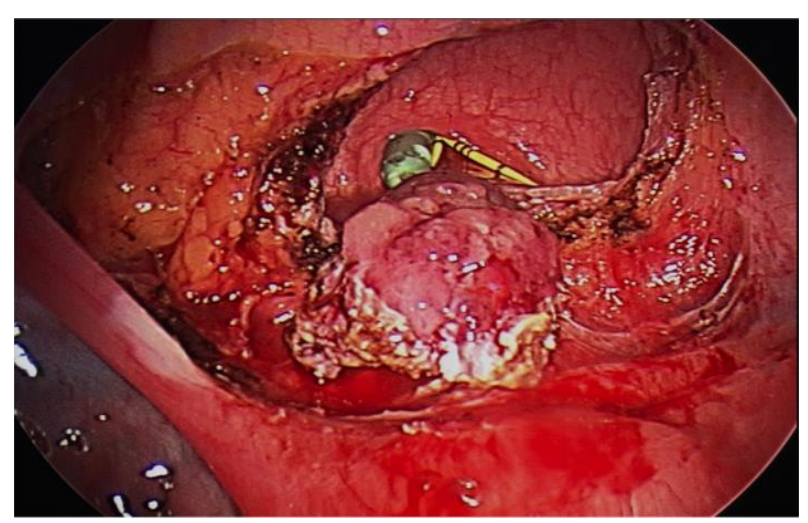

Figure 2: Laparoscopic view of bladder endometriosis.
First, the lesion was circumscribed and dissected free from the anterior uterine wall and cervix using a combination of hydrodissection and $\mathrm{CO}_{2}$ laser energy. Following laparoscopic mobilization of the lesion, the urologist circumscribed the endometriotic lesion through transurethral cystoscopy using a monopolar Collin's electrode. Final resection of the lesion was accomplished laparoscopically and it was removed through a $10 \mathrm{~mm}$ port. Repair of the cystostomy was carried using 2-vicryl suturing in a double-layer continuous fashion via the laparoscopic approach. The procedure was uneventful and the patient was discharged from hospital on the same day of operation, and the foley catheter was removed on postoperative day 10 , providing a healthy bladder on the CT cystogram, free from any fistula or leakage at the site of the repair. At six weeks follow-up, the patient reported a complete recovery and she was free of her previous symptoms of supra pubic pain and hematuria. The histopathology report confirmed nonmalignant endometriosis implant on the patient's bladder (Figure 3 ).

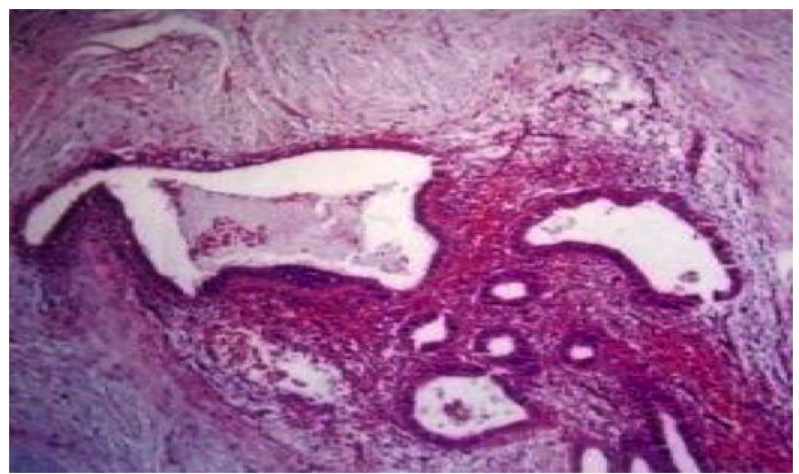

Figure 3: Histopathology slide.

\section{DISCUSSION}

The presence of endometrial tissue outside the uterus is known as endometriosis. It is believed to be the thirdleading cause of gynecological admissions in the United States. ${ }^{5}$ Deep infiltrative endometriosis (DIE) however, is a terminology given for the implantation of the endometrial glandular epithelium that penetrates the retroperitoneal space and invades the pelvic organs to a depth of $5 \mathrm{~mm}$ or more. The bladder is the most affected organ of the urinary tract system by DIE. ${ }^{2}$ Laparoscopic diagnosis of the visualized lesion, combined with histopathology, for any disease causing chronic pelvic pain has a sensitivity of $85.7 \% .{ }^{6}$ Moreover, transvaginal ultrasound (TVUS) remains the primary screening modality of choice for suspected cases of urinary tract endometriosis. ${ }^{7}$ In contrast with the limitations of TVUS in diagnosing deep posterior implants, MRI has demonstrated efficacy for both anterior and posterior implants, with $88 \%$ sensitivity and up to $99 \%$ specificity. ${ }^{2,8}$ It is crucial to emphasize that noninvasive imaging is preferred in the context of chronic pelvic pain, being better tolerated, safer and cheaper than surgery. ${ }^{6}$ 
Both hormonal-medical and surgical approaches for managing vesical endometriosis have been reported in the literature. It is worth noting that hormonal-medical therapy should not be prescribed alone, as the recurrence rate after discontinuation of the treatment is high. ${ }^{2}$ Partial cystectomy of bladder endometriosis can be achieved by open laparotomy or laparoscopic approach with or without utilization of robotic assistance. However, the laparoscopic approach has shown several advantages over the open procedure, namely, less bleeding, shorter operation time, shorter postoperative hospital stay and a general decrease in postoperative complications. ${ }^{9}$

It has been proposed that the laparoscopic approach should be reserved only for cases that allow free resection margin of at least $1-2 \mathrm{~cm}$, requiring the lesion to be far from the bladder neck, ureteral orifices and trigone. ${ }^{9}$ In a retrospective study by Chapron et al, which included 13 women who underwent laparoscopic excision for vesical lesions, with follow-up periods ranging from 4 to 77 months, the patients were clear from peri- and postoperative complications, and complete resolution of the symptoms was obtained. ${ }^{10}$

Transurethral surgery is another alternative that has been reported in the literature; however, if performed alone, it has several disadvantages, including high risk for bladder perforation, incomplete resection of the lesion, persistence of symptoms as well as high recurrence rate. Nevertheless, when transurethral surgery is combined with GnRH analogue hormonal therapy, a better control of the symptoms can be achieved, but with an estimated $25-35 \%$ recurrence rate. ${ }^{2}$ In this case, we performed a combined procedure of laparoscopic and transurethral partial cystectomy. However, this has not yet been adopted as a standard approach. A previous case report stated the advantages of performing a cystoscopic excision of the bladder lesion followed by laparoscopic intervention, which allowed better visualization of the transmural lesion that can be missed sometimes by the standard laparoscope. Furthermore, it granted a safer surgical margin for resection, especially for those nodules implanted on the trigone, bladder neck or near ureteral orifices. ${ }^{9}$ The present case indicates that the combined laparoscopic and cystoscopic approach is feasible, safe and results in good clinical outcomes as demonstrated by the absence of peri-operative complications as well as the complete resolution of symptoms.

\section{CONCLUSION}

A combination of laparoscopic and transurethral partial cystectomy may represent a safer alternative to the traditional laparoscopic approach for bladder endometriosis; we recommend further studies to explore the adoption of this procedure as the new standard technique.

\section{Funding: No funding sources \\ Conflict of interest: None declared \\ Ethical approval: Not required}

\section{REFERENCES}

1. Sener A, Chew B, Duvdevani M, Brock G, Vilos G, Pautler S. Combined transurethral and laparoscopic partial cystectomy and robot-assisted bladder repair for the treatment of bladder endometrioma. J Minim Invasive Gynecol. 2006;13(3):245-8.

2. Maccagnano C, Pellucchi F, Rocchini L, Ghezzi M, Scattoni V, Montorsi F et al. Diagnosis and treatment of bladder endometriosis: State of the Art. Urol Int. 2012;89(3):249-58.

3. Bogart L, Berry S, Clemens J. Symptoms of interstitial cystitis, painful bladder syndrome and similar diseases in women: a systematic review. J Urol. 2007;177(2):450-6.

4. Vilos G, Vilos A, Abu-Rafea B, Hollett-Caines J, Nikkhah-Abyaneh Z, Edris F. Three simple steps during closed laparoscopic entry may minimize major injuries. Surg Endosc. 2008;23(4):758-64.

5. Missmer SCramer D. The epidemiology of endometriosis. Obstet Gynecol Clin North Am. 2003;30(1):1-19.

6. Tirlapur S, Daniels J, Khan K. Chronic pelvic pain. Curr Opin Obstet Gynecol. 2015;27(6):445-8.

7. Mettler L, Gaikwad V, Riebe B, Schollmeyer T. Bladder Endometriosis: Possibility of Treatment by Laparoscopy. JSLS. 2008;12:162-5.

8. Balleyguier C, Chapron C, Dubuisson J, Kinkel K, Fauconnier A, Vieira M, et al. Comparison of Magnetic Resonance Imaging and Transvaginal Ultrasonography in Diagnosing Bladder Endometriosis. J Am Assoc Gynecol Laparosc. 2002;9(1):15-23.

9. Walid MHeaton R. Laparoscopic partial cystectomy for bladder endometriosis. Arch Gynecol Obstet. 2008;280(1):131-5.

10. Chapron C, Dubuisson JB , Jacob S , Fauconnier A, Da Costa Vieira M. Laparoscopy and bladder endometriosis. Gynecologie, Obstetrique and Fertilite. 2000;28(3):232-7.

Cite this article as: Algreisi $\mathrm{F}$, Badr $\mathrm{H}$, Alturkistani D, Vilos G. Combined transurethral and laparoscopic partial cystectomy for the treatment of bladder endometriosis. Int J Reprod Contracept Obstet Gynecol 2017;6:1122-4. 